\title{
Trajetória Hipotética de Aprendizagem como recurso para a formação de professores
}

\author{
Hypothetical Learning Trajectory as a resource for teacher education
}

\author{
Julio Cezar Rodrigues de Oliveira ${ }^{1}$ \\ Pamela Emanueli Alves Ferreira ${ }^{2}$
}

\begin{abstract}
Resumo
O objetivo deste artigo é apresentar um ensaio teórico e discutir aspectos de uma Trajetória Hipotética de Aprendizagem (THA) com vistas a subsidiar práticas de professores de Matemática em formação inicial ou continuada. $\mathrm{O}$ estudo se trata de uma pesquisa qualitativa e, à luz de pressupostos teóricos de Martin Simon busca-se argumentar a respeito da elaboração de THAs como um recurso estratégico metodológico que possibilita aos docentes em formação (ou em exercício) organizarem seus planejamentos escolares a partir de um processamento hipotético. Para atingir estes objetivos são apresentados: aspectos teóricos da THA; uma THA sobre o ensino de Logaritmos; uma discussão a respeito do processo de sua elaboração como estratégia de formação. Como resultado infere-se que as THAs oportunizam aos docentes em formação analisarem aspectos que delimitam suas práticas de modo articulado, se envolverem em um processo de reflexão e tomada de decisão sobre seu exercício profissional. As análises e reflexões se amparam no argumento de a THA se revelar como um potencial recurso para a formação docente por apresentar carácter instrumentalizador, flexível, interativo, previsor, problematizador, dinâmico.
\end{abstract}

Palavras-chave: Educação Matemática; Formação de professores de matemática; Trajetórias Hipotéticas de Aprendizagem.

\begin{abstract}
The purpose of this article is to present a theoretical essay and discuss aspects of a Hypothetical Learning Trajectories (HLT) with the goal to subsidizing the practices of mathematics teachers in pre-service or in-service education. The study is a qualitative research and, in the light of Martin Simon's theoretical assumptions, we seek to argue about the elaboration of HLT as a strategic methodological resource that enables pre-service or inservice teachers to organize their school plans based on hypothetical processing. To achieve these objectives, the following are presented: theoretical aspects of HLT; an HLT on teaching Logarithms; a discussion about the process of its development as a education strategy. As a result, it is inferred that HLTs provide opportunities for teachers education to analyze aspects that delimit their practices in an articulated way, to get involved in a process of reflection and decision-making about their professional practice. The analyzes and reflections are
\end{abstract}

Submetido em: 30/10/2020 - Aceito em: 01/02/2021 - Publicado em: 29/05/2021

${ }^{1}$ Mestre em Ensino de Ciências e Educação Matemática pela Universidade Estadual de Londrina. Professor da Educação Básica da Rede Estadual do Paraná, Toledo, Brasil. E-mail: julioeconomit@hotmail.com. ORCID: https://orcid.org/0000-0002-1383-2477

${ }^{2}$ Doutora em Ensino de Ciências e Educação Matemática pela Universidade Estadual de Londrina. Professora da Universidade Estadual de Londrina, Londrina, Brasil. E-mail: pam@uel.br. ORCID: http://orcid.org/0000$\underline{0002-9420-8536}$ 
DOI: $10.20396 /$ zet.v29i00.8661816

supported by the argument that THA is revealed as a potential resource for teacher training because it has an instrumental, flexible, interactive, predictive, problematic and dynamic character.

Keywords: Mathematics Education; Mathematics teacher education; Hypothetical Learning Trajectories.

\section{Introdução}

A opção pela apresentação de uma Trajetória Hipotética de Aprendizagem (THA) neste artigo deu-se porque se acredita que ela tem potencial para que o professor possa ir além da elaboração de um plano de aula e reflita a respeito de suas ações e das possíveis ações dos estudantes em sala de aula. Uma THA pode ser brevemente definida como um planejamento que o professor faz com base em suas experiências anteriores enquanto professor e enquanto estudante, ao considerar, por exemplo, as possíveis dúvidas que os estudantes podem apresentar em uma aula de determinado conteúdo. Na THA o professor leva em conta três elementos: os objetivos que ele tem para a aprendizagem; o plano que ele elabora com as atividades de aprendizagem; e o processo hipotético de aprendizagem, que descreve como o pensamento e a compreensão dos estudantes evoluirão no contexto das atividades de aprendizagem (Simon, 1995).

Nesse sentido, acredita-se que o presente estudo é pertinente, pois representa uma alternativa para o ensino de Matemática, em especial para a formação docente, por meio da elaboração de Trajetórias Hipotéticas de Aprendizagem (THAs), as quais podem fornecer subsídios tanto para a formação profissional quanto para o exercício docente.

\section{Procedimentos Metodológicos}

O presente estudo é fruto de uma pesquisa qualitativa e, à luz dos pressupostos teóricos de Simon (1995) e demais autores busca-se apresentar um ensaio teórico com vistas a argumentar a respeito da elaboração de THAs como um recurso estratégico metodológico que possibilita aos docentes em formação organizarem seus planejamentos escolares a partir de um processamento hipotético. Para atingir estes objetivos são apresentados:

- aspectos teóricos a respeito de Trajetória Hipotética de Aprendizagem de Simon (1995);

- uma proposta de THA sobre ensino de $\operatorname{logaritmos}^{3}$ na perspectiva da Resolução de Problemas;

- uma discussão a respeito da elaboração de THAs como estratégia de formação.

Com esse trabalho pretende-se discutir as seguintes questões: as THAs oportunizam aos docentes em formação analisarem aspectos que delimitam suas práticas de modo articulado, se envolverem em um processo de reflexão e tomada de decisão sobre demandas

\footnotetext{
${ }^{3}$ Não se tem como objetivo, neste artigo, discutir especificamente o ensino de Logaritmos ou a aplicação da THA que é apresentada. Ela foi utilizada apenas como contexto para motivar a discussão.
} 
DOI: $10.20396 /$ zet.v29i00.8661816

de seu exercício profissional; a THA pode se configurar como um recurso instrumentalizador, flexível, interativo, previsor, problematizador, dinâmico; a THA se revela como um potencial instrumento o qual pode oportunizar o desenvolvimento do conhecimento de professores de matemática e de formadores de professores.

Como resultado, acredita-se que a elaboração e exploração de THAs por meio da Resolução de Problemas têm potencial para o ensino de matemática e para a formação de professores, pois fornece subsídios tanto teóricos como práticos para que o professor trabalhe com a matemática nessa perspectiva.

\section{Aspectos teóricos a respeito de Trajetória Hipotética de Aprendizagem de Simon (1995)}

Algumas dissertações de Mestrado Profissional em Ensino de Matemática da PUCSão Paulo apontam que as Trajetórias Hipotéticas de Aprendizagem na perspectiva de Simon (1995) representam uma alternativa para o professor planejar sua atuação em sala de aula, tais como Angiolin (2009), Barbosa (2009), Lima (2009), Luna (2009), Menotti (2014), Mesquita (2009), e Rosenbaum (2010).

$\mathrm{Na}$ tentativa de tentar responder à questão: "como os professores de matemática podem promover a construção de ideias matemáticas que a comunidade de matemáticos levou milhares de anos para desenvolver?". Simon (1995) considera o planejamento do professor como um fator essencial, uma vez que, dentre suas responsabilidades, está o planejamento. Brousseau (1987) afirma que, em seu planejamento, parte do papel do professor envolve tornar ideias matemáticas não contextualizadas que precisam ser ensinadas e embuti-las em um contexto para que os estudantes possam investigá-la. Tal contexto deve apresentar um significado para os estudantes, possibilitando que eles resolvam problemas nesse contexto, e a sua solução pode envolver uma ideia matemática nova a ser aprendida. A resolução para o problema pode não ser única.

Uma das responsabilidades fundamentais de professores é a de entender como se constitui o conhecimento matemático de seus estudantes e como articular seu método de ensino com a natureza desse conhecimento matemático. Nesse sentido, Simon (1995) examina o papel de diferentes aspectos do conhecimento dos professores e explora o desafio contínuo e inerente de integrar os objetivos do professor e a aprendizagem dos estudantes por meio do que chamou de Trajetória Hipotética de Aprendizagem.

Simon (1995) apresentou a trajetória hipotética de aprendizagem por meio do Ciclo de Ensino de Matemática, que ele desenvolveu como um modelo do inter-relacionamento cíclico de aspectos que envolvem o conhecimento do professor, seu pensamento e a tomada de decisões com relação ao seu planejamento. 
Com base em três episódios de seu estudo ${ }^{4}$ realizado sobre o ensino de área, Simon (1995) afirmou que dois fatores foram considerados para o objetivo e a estrutura da lição: a compreensão matemática do professor e as hipóteses do professor a respeito do conhecimento dos estudantes. O autor justifica que utiliza o termo "hipótese" porque o professor não tem acesso direto ao conhecimento dos estudantes, mas pode inferir a natureza da compreensão dos estudantes a partir das interpretações dos comportamentos que eles apresentam. Para Oliveira et al. (2014), com base em Simon (1995) isso implica que o professor pode comparar a sua compreensão de um conceito particular a partir da sua construção de entendimentos dos estudantes hipoteticamente, mas não é possível que ele conheça de antemão os entendimentos reais dos estudantes.

A partir dessas considerações, Simon (1995) apresenta o objetivo de aprendizagem do professor como o ponto de partida para a elaboração de uma trajetória hipotética de aprendizagem. De acordo com o autor, o uso da expressão "trajetória hipotética de aprendizagem" é para se referir à

previsão do professor como um caminho pelo qual a aprendizagem pode ocorrer. É hipotético porque a trajetória real de aprendizagem não é conhecida previamente. Ela caracteriza uma tendência esperada. A aprendizagem individual dos estudantes ocorre de forma idiossincrática, embora frequentemente em caminhos similares. É assumido que uma aprendizagem individual tem alguma regularidade (cf. Steffe, et al., 1983, p. 118), que a sala de aula limita a atividade matemática frequentemente de formas previsíveis, e que muitos estudantes na mesma sala podem se beneficiar da mesma tarefa matemática. Uma trajetória hipotética de aprendizagem fornece ao professor uma análise racional para escolher um projeto instrucional particular; assim, eu tomo as minhas decisões baseado nas minhas melhores suposições de como a aprendizagem pode acontecer (Simon, 1995, p. 135, tradução nossa).

A expressão "trajetória hipotética de aprendizagem" é utilizada com o objetivo de enfatizar aspectos do pensamento do professor que estão fundamentados em uma perspectiva construtivista e são comuns tanto ao planejamento antecipado e à tomada de decisões espontânea (Simon, 1995).

O autor justifica sua escolha pela palavra "trajetória" para referir-se a um caminho, e faz uma analogia a uma viagem, considerando que se pretenda fazer uma viagem pelo mundo, e não se pretende viajar aleatoriamente, mas também não planejou um itinerário a ser seguido. Com esse objetivo, busca-se o máximo de informações a respeito de cada lugar que se pretende visitar, elaborando um plano.

A princípio, pode-se ter toda a viagem planejada ou apenas parte dela. No início da viagem, segue-se o plano, mas no decorrer do trajeto, devido às condições que se encontra, é necessário ajustar constantemente esse plano de viagem. Dessa forma pode ser que se mude a ordem pela qual os lugares serão visitados ou fique-se por mais tempo em determinados

\footnotetext{
${ }^{4} \mathrm{Na}$ pesquisa desenvolvida por Simon, os dados foram coletados em uma sala de aula experimental com 25 alunos. O pesquisador acompanhou um professor de Matemática em tarefas que envolvem o conceito de área, e após a análise de dados coletados, ele trabalhou em uma fundamentação teórica com o intuito de formular uma pedagogia da Matemática.
} 
DOI: $10.20396 /$ zet.v29i00.8661816

lugares e menos tempo em outros, podendo até deixar de visitar alguns lugares e visitar outros que não havia planejado. Enfim, o caminho pelo qual se viaja é a "trajetória", e o caminho que se havia planejado é a "trajetória hipotética" (Simon, 1995).

A noção de THA na perspectiva de Simon não impõe que o professor sempre busque um objetivo por vez, ou que apenas uma trajetória deve ser considerada, mas vale salientar a importância de se ter um objetivo, fazer uma análise racional para a tomada de decisões do professor, e a natureza hipotética de tal pensamento (Oliveira, 2014, p. 4).

Simon (1995) considera que uma trajetória hipotética de aprendizagem é composta por três componentes:

1) o objetivo de aprendizagem, que define uma direção para o planejamento do professor;

2) o plano que o professor elabora com as atividades de aprendizagem;

3) o processo hipotético de aprendizagem, que apresenta uma previsão de como o pensamento e a compreensão dos estudantes evoluirão no contexto das atividades de aprendizagem.

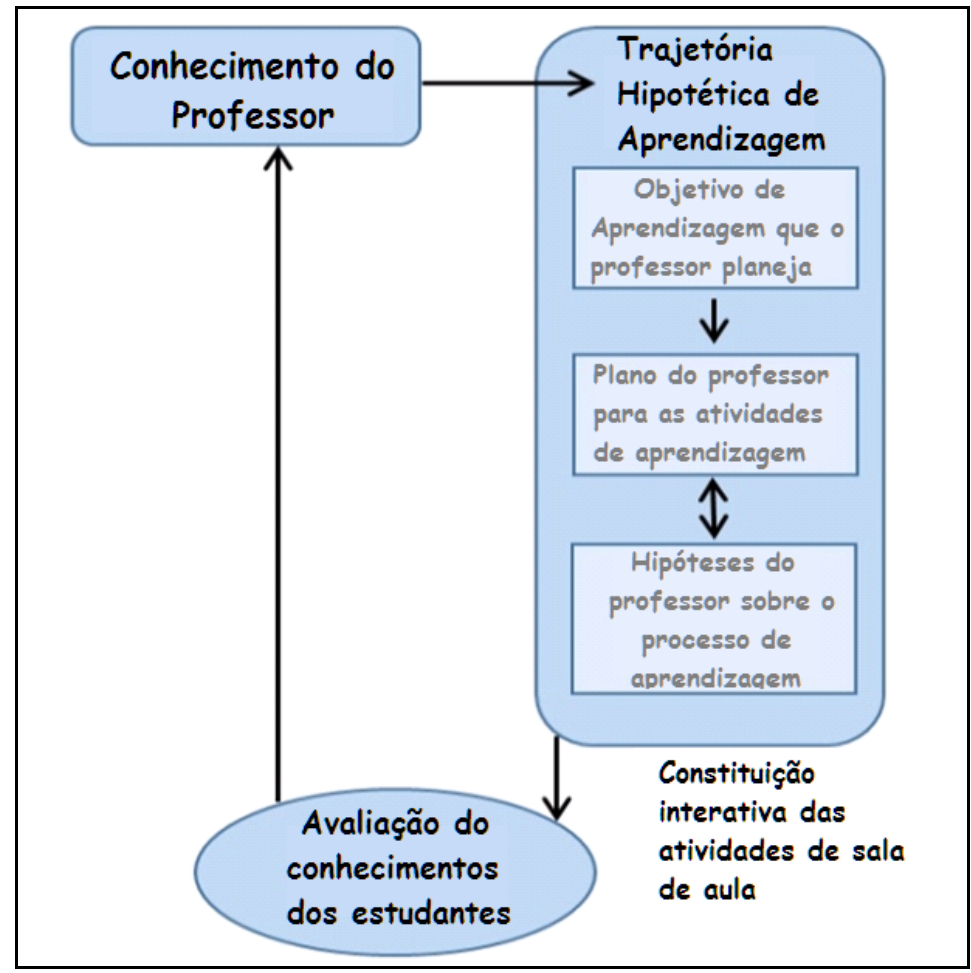

Figura 1 - Ciclo de Ensino de Matemática (abreviado)

Fonte: dos autores. Traduzido e adaptado de Simon (1995)

A partir de seu conhecimento, o professor considera os objetivos que planeja alcançar por meio de um plano de atividades, e nesse plano apresenta suas hipóteses sobre como será a 
aula que planejou. Gómez, González e Lupiáñez (2007) assumem que o professor pode escolher um objetivo de aprendizagem específico para o qual ele está planejando a aula. Esse objetivo constitui uma estrutura de referência que delimita as condições e os procedimentos que o professor espera desenvolver com o objetivo de formular suas hipóteses sobre o processo de aprendizagem dos estudantes. A informação da qual o professor dispõe precisa ser organizada em um processo sistemático para tentar alcançar os objetivos que ele estabeleceu.

Um objetivo de aprendizagem é uma noção complexa. Se o professor quer planejar tarefas para promover a realização desse objetivo por seus alunos, então é necessário caracterizá-lo de tal forma que ele pode conjecturar como e em que medida uma tarefa (ou uma sequência de tarefas) pode contribuir para sua realização (Gómez, González \& Lupiáñez, 2007, p. 3, tradução nossa).

Uma vez definido o objetivo de aprendizagem, a elaboração de uma trajetória hipotética de aprendizagem envolve a geração de um processo de aprendizagem hipotético de um conjunto particular de tarefas. Simon e Tzur (2004) apresentam algumas questões que o professor pode pensar para a criação desse processo: "qual tarefa, atualmente disponível para os estudantes, pode ser a base para que eles consigam alcançar os objetivos de aprendizagem?" A partir dessa questão, o professor pode buscar em seus materiais qual tarefa traz essas possibilidades para que ele alcance seus objetivos.

Enquanto os objetivos de aprendizagem fornecem uma direção para a elaboração da trajetória hipotética de aprendizagem, a seleção de tarefas e as hipóteses sobre o processo da aprendizagem dos estudantes são interdependentes. As tarefas são selecionadas com base nas hipóteses que o professor tem quanto ao processo de aprendizagem, e a hipótese do processo de aprendizagem está baseada nas tarefas que estarão envolvidas (Simon \& Tzur, 2004). Nesse sentido, algumas suposições subjazem a essas ideias

1. A elaboração de uma trajetória hipotética de aprendizagem está baseada na compreensão do conhecimento atual dos estudantes envolvidos.

2. Uma trajetória hipotética de aprendizagem é um veículo para o planejamento da aprendizagem de determinados conceitos matemáticos.

3. As tarefas matemáticas fornecem ferramentas para promover a aprendizagem de um conceito matemático particular e são, portanto, um elemento chave do processo de ensino.

4. Devido à natureza hipotética e inerentemente incerta desse processo, o professor está regularmente envolvido em modificar cada aspecto da trajetória hipotética de aprendizagem (Simon \& Tzur, 2004, p. 93).

Uma vez selecionadas as tarefas para a trajetória hipotética de aprendizagem, o professor pode considerar suas hipóteses para o processo de aprendizagem mais detalhadamente. Para tanto, ele pode considerar as possíveis dúvidas que os estudantes apresentem enquanto tentam resolver as tarefas. Ao levantar essas dúvidas, o professor também antecipa as possíveis respostas para que os estudantes consigam compreender os detalhes que os deixaram confusos. 
DOI: $10.20396 /$ zet.v29i00.8661816

Depois de estabelecer o(s) objetivo(s) de aprendizagem e selecionar as tarefas, o professor avalia o trabalho que desenvolveu e tem a possibilidade de reformular sua trajetória hipotética de aprendizagem (Simon, 1995). Na Figura 1, o diagrama indica que a avaliação dos estudantes é contínua e pode trazer adaptações para o conhecimento do professor, o que pode conduzir a uma trajetória hipotética de aprendizagem nova ou modificada.

A elaboração de uma trajetória hipotética de aprendizagem antes do ensino na sala de aula é um processo pelo qual o professor desenvolve um plano de atividades para ser realizado em sala de aula por seus estudantes. Contudo, ao interagir com os estudantes e observá-los, o professor e os estudantes constituem uma experiência, que pela sua natureza social, é diferente daquela antecipada pelo professor. Nesse contexto, as ideias do professor sobre o conhecimento dos estudantes podem modificar-se e ele tem a possibilidade de modificar a trajetória hipotética de aprendizagem que havia elaborado anteriormente (Simon, 1995).

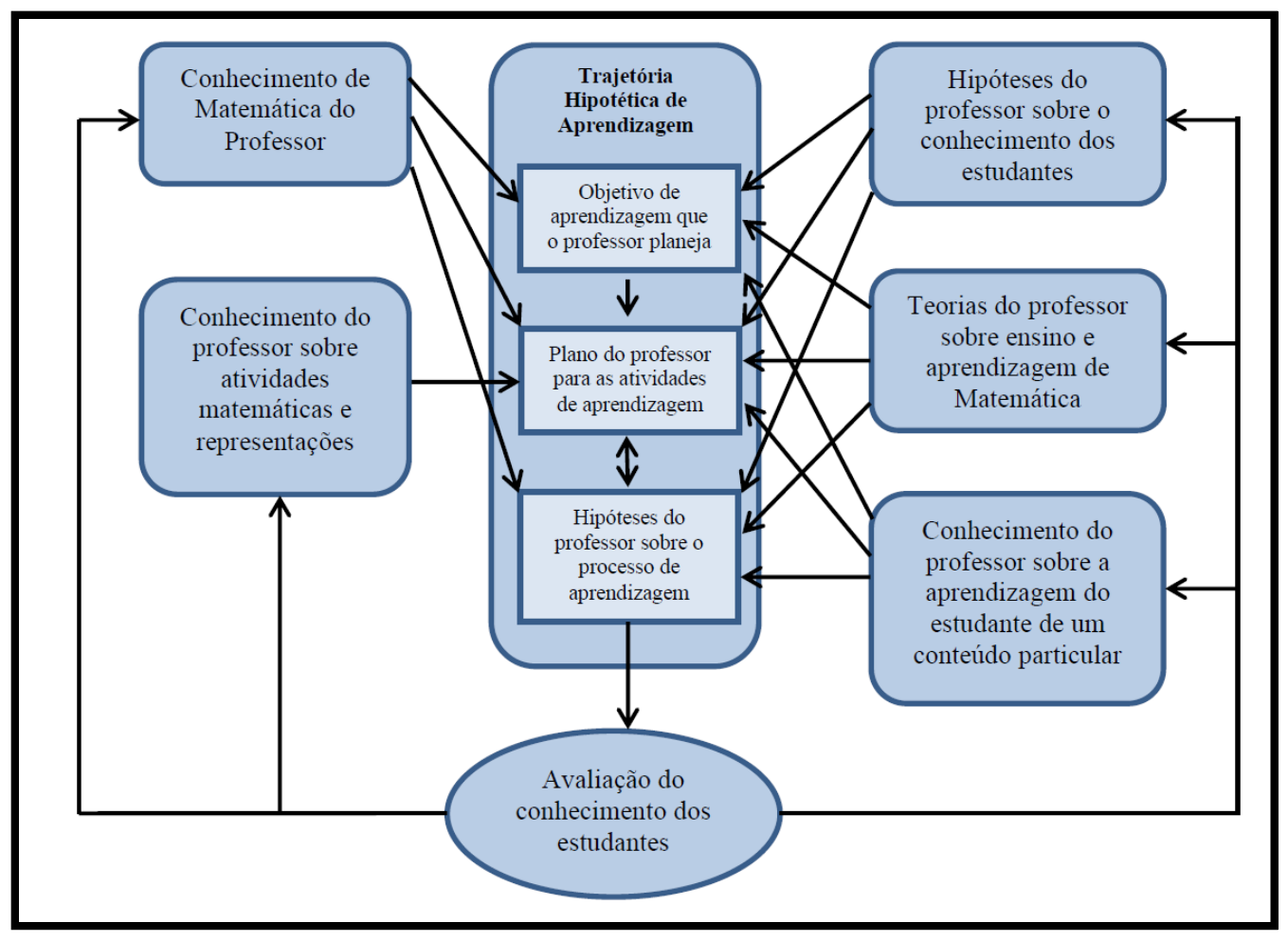

Figura 2 - Ciclo de Ensino de Matemática

Fonte: dos autores. Traduzido e adaptado de Simon (1995)

A Figura 2 descreve as relações entre os domínios do conhecimento do professor, a trajetória hipotética de aprendizagem e as interações com os estudantes. Simon (1995) explica a figura

Começando pelo topo do diagrama, o conhecimento de matemática do professor em interação com as hipóteses sobre o conhecimento matemático dos estudantes, contribuem para a identificação de um objetivo de aprendizagem. Esses domínios de conhecimento, o objetivo de aprendizagem, o conhecimento de atividades matemáticas e representações do professor, seu conhecimento sobre a aprendizagem 
DOI: $10.20396 /$ zet.v29i00.8661816

dos estudantes de um conteúdo particular, bem como as concepções do professor sobre ensino e aprendizagem [...] contribuem para o desenvolvimento de atividades de aprendizagem e um processo de aprendizagem hipotético. A modificação da trajetória hipotética de aprendizagem não é algo que ocorre apenas durante o processo de planejamento entre as aulas. $\mathrm{O}$ professor está continuamente engajado para ajustar a trajetória de aprendizagem que ele conjecturou para melhor refletir seu conhecimento aprimorado. Às vezes, pequenos ajustes são necessários, embora algumas vezes toda a essência da lição deve ser descartada e substituída por outra mais apropriada. Independentemente da extensão da modificação, mudanças devem ser feitas em qualquer ou em todos os três componentes da trajetória hipotética de aprendizagem: o objetivo, as atividades, ou o processo hipotético de aprendizagem (Simon, 1995, p. 138, tradução nossa).

De acordo com Gómez, González e Lupiáñez (2007), o conhecimento do professor, sua experiência e a literatura disponível são as fontes básicas para que ele possa elaborar uma trajetória hipotética de aprendizagem que apoie seu planejamento.

Ao elaborar uma trajetória hipotética de aprendizagem, o professor planeja possíveis situações e rotas pelas quais a aprendizagem pode ocorrer no contexto de tarefas particulares. Quando uma tarefa matemática não é suficiente para que o professor tenha indícios de que os estudantes aprenderam da forma como havia planejado, ele ajusta sua trajetória hipotética de aprendizagem, geralmente modificando a tarefa e às vezes alterando a interpretação que ele tinha dos conceitos dos estudantes nas quais sua trajetória está baseada (Simon \& Tzur, 2004), ou, também, propondo novas tarefas em diferentes contextos. De acordo com Steffe (2004)

a construção de trajetórias hipotéticas de aprendizagem dos estudantes é um dos problemas mais difíceis, porém urgentes que a educação matemática enfrenta atualmente. É também um dos problemas mais empolgantes porque é nela que podemos construir uma compreensão da matemática dos estudantes e como nós professores podemos afetar de forma rentável essa matemática (Steffe, 2004, p. 130, tradução nossa).

Nesse sentido, corrobora-se com Pires (2009, p. 164) ao afirmar que "os jovens professores precisam de conhecimentos sobre os saberes dos alunos, para gerar trajetórias hipotéticas de aprendizagem e análises conceituais para que possam ensinar Matemática".

Alguns estudos têm apontado para a elaboração de Trajetórias Hipotéticas de Aprendizagem como oportunidade para a formação do professor de Matemática (Ivars et al., 2018; Sztajn et al., 2012; Wilson et al., 2014; Wilson et al., 2017).

Em Sztajn et al. (2012), os autores argumentam que as Trajetórias de Aprendizagem utilizadas na formação fornecem quatro estruturas para o ensino da matemática: o conhecimento matemático para o ensino, análise de tarefas, discurso como facilitador práticas de ação e avaliação formativa. Eles apresentam dois esquemas na tentativa de: (1) conceitualizar o ensino na perspectiva das Trajetórias de Aprendizagem; (2) definir componentes da instrução baseados em Trajetórias de Aprendizagem. Neste segundo esquema, o objetivo é de associar, os elementos das THA com os quatro domínios do 
"Conhecimento matemático para o ensino" (Ball \& Bass, 2003; Ball, Thames \& Phelps, 2008).

Na pesquisa de Wilson et al. (2014) é relatada uma experiência na qual os autores examinam o design de um programa criado para apoiar a aprendizagem de professores em uma trajetória de aprendizagem realizando uma reinterpretação a partir da perspectiva do conhecimento matemático para o ensino (Ball, Thames, \& Phelps, 2008). Os resultados indicaram que as tarefas de aprendizagem profissional com foco no conhecimento do conteúdo pedagógico presente nas trajetórias de aprendizagem permitem a aprendizagem do professor sobre o "conhecimento do conteúdo".

Ivars et al. (2018) inferem que o uso de uma trajetória de aprendizagem hipotética como um guia para interpretar o pensamento matemático dos alunos pode melhorar o discurso dos professores em formação. No estudo destes autores, vinte e nove professores em formação participaram de um ambiente de aprendizagem em que tiveram que interpretar o pensamento dos alunos sobre o conceito de fração usando uma trajetória de aprendizagem hipotética como guia. Essa ação de formação ajudou os professores a desenvolver um discurso mais detalhado ao interpretar o pensamento matemático dos alunos, aprimorando suas habilidades de "perceber", as quais estavam ligadas ao conhecimento do conteúdo matemático dos professores em formação, segundo estes autores.

$\mathrm{Na}$ tentativa de dialogar com esses pressupostos teóricos a respeito da THA como elemento formador, na seção seguinte apresenta-se uma primeira versão de uma Trajetória Hipotética de Aprendizagem para o ensino de logaritmos na perspectiva da Resolução de Problemas, considerando algumas possibilidades para a abordagem desse conteúdo. A partir da elaboração dessa trajetória, na seção posterior, buscar-se-á refletir a respeito dessa atividade como instrumento de formação docente.

\section{Uma THA sobre o ensino de Logaritmos na perspectiva da Resolução de Problemas.}

Nesta seção propõe-se a apresentação sob alguns recortes" de uma "trajetória hipotética de aprendizagem", para o ensino de logaritmos e suas propriedades operatórias a partir de um problema. A THA foi elaborada na perspectiva da Resolução de Problemas, de Onuchic e Allevato (2011), para que pudesse ser utilizada para introduzir o conceito de logaritmo e de suas propriedades operatórias no Ensino Médio. Como o objetivo deste artigo não é discutir a THA em si, mas sim que características do seu processo de elaboração são potenciais e caracterizam a THA como um instrumento que pode oportunizar o desenvolvimento do conhecimento de professores que ensinam matemática, optou-se por

\footnotetext{
${ }^{5}$ Esse recorte é justificado pela quantidade limite de páginas para este artigo, tomado o cuidado de não interferir na qualidade do mesmo, considerando a discussão que se pretende promover.
} 
realizar alguns "recortes" da THA original nessa seção para subsidiar as discussões que serão promovidas na seção seguinte ${ }^{6}$.

Para a elaboração da THA, foi considerado o conteúdo logaritmos e suas propriedades operatórias por se tratar de um conteúdo do Ensino Médio, como parte do tópico funções, e em específico relacionado às funções exponenciais e logarítmicas. No entanto, o ensino de logaritmos ainda é, de forma geral, desenvolvido por meio da apresentação de definições, propriedades, e pela resolução de exercícios propostos em uma lista, para que na sequência sejam trabalhados problemas que envolvem esses conteúdos. A intenção é trabalhar em um sentido inverso, ou seja, apresentar um problema que pudesse ser solucionado por meio dos logaritmos e suas propriedades operatórias, e dessa forma, os estudantes compreenderem a importância do estudo desse conteúdo ao resolver um problema que o aborda.

Na THA proposta a seguir, não há uma divisão linear de todas as etapas de uma aula apresentadas no roteiro proposto por Onuchic e Allevato (2011) e inspirado nas etapas de resolução de problemas de Polya (1994). Destaca-se a etapa da resolução do problema para descrever uma possível solução (hipotética) que os estudantes podem apresentar, incluindo possíveis dúvidas no decorrer da resolução, conforme sugerido por Simon (1995), como as hipóteses do professor sobre o processo de aprendizagem. Na THA original, enfatiza-se também a formalização do conteúdo no decorrer da aula, uma vez que a resolução do problema e a formalização representam aspectos essenciais da estratégia metodológica de ensino adotada. Justifica-se essa escolha pela razão de ser "uma proposta", e nesse caso, há algumas etapas que estão descritas de forma detalhada e outras nem tanto devido à dependência/flexibilidade de como essa proposta pode ser implementada.

A THA apresentada na íntegra em Oliveira (2015) tem como finalidade discutir cada uma das etapas desde a introdução do problema até a sua formalização segundo a perspectiva da Resolução de Problemas. Ela apresenta o seguinte formato: (1) Apresentação da Atividade Proposta; (2) Objetivos para a Aprendizagem; (3) Compreendendo o Problema; (4) Estabelecendo um plano; (5)Executando o plano; (6) Retrospecto; (7) Formalização do conteúdo: conhecendo os logaritmos; (8) Estudando as consequências da definição; (9) Explorando algumas propriedades dos Logaritmos; (10) Consequências da Propriedade de Mudança de Base; (11) Sistemas de Logaritmos; (12) Voltando ao problema. O recorte para a discussão deste artigo vai contar com a apresentação dos itens de (1) à (6) apresentados a seguir.

\section{(1) Apresentação da Atividade Proposta}

Para começar a trabalhar com esta THA, sugere-se que o professor contextualize a situação-problema que será apresentada, conversando sobre recursos naturais, sua escassez e o que tem sido feito para que eles sejam aproveitados da melhor forma possível, considerando os meios de produção de alimentos utilizados atualmente. Na sequência, é

\footnotetext{
${ }^{6}$ A trajetória na íntegra pode ser obtida em Oliveira (2015).
} 
DOI: $10.20396 /$ zet.v29i00.8661816

possível relacionar a discussão com a população mundial, questionando se os estudantes sabem em que proporção população mundial cresce a cada ano, qual o percentual médio de crescimento anual mundial, para então ser apresentada a seguinte situação-problema para a turma:

\section{Até quando o planeta pode nos sustentar? ${ }^{7}$}

Estima-se que $1350 \mathrm{~m}^{2}$ de terra sejam necessários para fornecer alimento para uma pessoa. Admite-se, também, que há $28 \times 1350$ bilhões de $\mathrm{m}^{2}$ de terra arável no mundo e que, portanto, uma população máxima de 28 bilhões de pessoas pode ser sustentada, se não forem exploradas outras fontes de alimento. A população mundial, no início de 2013, foi estimada em aproximadamente 7 bilhões de habitantes. Considerando que a população continua a crescer, a uma taxa de aproximadamente $2 \%$ ao ano, determine em quantos anos, a partir de 2013, a Terra teria a máxima população que poderia ser sustentada.

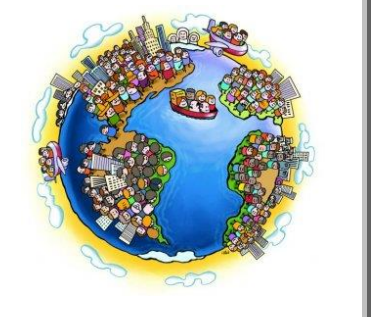

(Adaptado de: Unb - 1996)

Fonte: http://www.estadao.com.br/ - acesso em 09/03/2014

\section{Figura 3 - Enunciado do Problema}

A leitura do enunciado pode ser feita individual e coletivamente, e o professor pode pedir que os estudantes expliquem o que entenderam a respeito do problema. Caso eles apresentem alguma dúvida com relação ao significado de alguma palavra, eles podem conferir em um dicionário para compreender esse significado.

O problema pode ser proposto aos estudantes para que eles resolvam individualmente ou em pequenos grupos, e o professor acompanha as resoluções desses grupos nas carteiras, verificando se os estudantes têm dúvidas ao resolvê-lo e levantando questionamentos para que eles consigam refletir a respeito de suas dúvidas e compreender como podem elaborar estratégias para a resolução desse problema. Na sequência, são apresentadas uma resolução desse problema e dúvidas que os estudantes podem apresentar ao tentar resolvê-lo, considerando as quatro fases apresentadas no modelo de Polya (1994).

\section{(2) Objetivos para a Aprendizagem}

- Resolver um problema situado em um contexto com referência na realidade.

- Utilizar conceitos de proporcionalidade para estabelecer relações entre as diferentes grandezas.

- Descrever a situação-problema em um contexto matemático.

- Desenvolver procedimentos relativos ao uso de Logaritmos e suas propriedades operatórias para resolver o problema.

\footnotetext{
${ }^{7}$ Ao apresentar esse problema para os estudantes, o professor pode ou não apresentar os seguintes dados: (ln $1,02=0,02$ e $\ln 2=0,70)$.
} 


\section{(3) Compreendendo o Problema}

Considerando o enunciado apresentado, espera-se que os estudantes sintam-se desafiados a buscar uma resolução que responda à pergunta do problema. Para tanto, o professor pode investigar com os estudantes quais são as informações relevantes que podem ser elencadas:

A população mundial em 2013 era de 7 bilhões de habitantes, ou, em notação científica, era de $7.10^{9}$ bilhões de habitantes, que será considerada como população inicial $(\mathrm{P}(0))$.

Possível Dúvida: os estudantes podem questionar com relação à notação utilizada.

Por que utilizar $\mathrm{P}(0)$ como população inicial?

O professor pode justificar essa escolha ao afirmar que essa é a população considerada no tempo inicial, que nesse caso é o ano de 2013, ou também pode reelaborar a pergunta aos estudantes esperando que eles cheguem a essa conclusão. Outra possibilidade é deixar que os estudantes escolham como utilizar a notação para a população de 2013, ao que eles podem escolher a notação de $\mathrm{P}(2013)$. Na discussão que se apresenta nessa THA, considera-se $\mathrm{P}(0)$, mas nada impede que os estudantes utilizem outra notação para representar a população inicial.

\section{(4) Estabelecendo um plano}

Ao pensar sobre o crescimento populacional, o enunciado afirma que a população continua a crescer a uma taxa de $2 \%$ ao ano, logo se sabe que o fator pelo qual se multiplica a população de cada ano é $102 \%=1,02$ para descobrir a população do ano seguinte.

Possível Dúvida: os estudantes podem apresentar dúvidas com relação à representação da porcentagem.

Mas podemos afirmar que $102 \%=1,02$ ? Por quê?

O professor pode retomar o significado da porcentagem ao questioná-los sobre as representações de 102\%. Ao pensar em $102 \%$ de um valor qualquer, pode-se utilizar a porcentagem na representação fracionária: $102 \%=\frac{102}{100}$. E ao dividir 102 por 100, obtém-se 1,02. Além disso, o professor pode aproveitar essa questão para discutir com os estudantes a respeito da população dos anos seguintes, sugerindo a elaboração de um quadro.

Nesse quadro, será utilizada a letra $t$ para representar o tempo em anos, considerando que o ano de 2013 é o ano inicial, no qual $t=0$. Então, para determinar a população dos anos seguintes, pode-se calcular da seguinte forma: 
DOI: $10.20396 /$ zet.v29i00.8661816

Quadro 01: População em função do Tempo

\begin{tabular}{|c|c|c|}
\hline Ano & $\mathbf{t}$ (em anos) & P (população no ano t) \\
\hline 2013 & 0 & $P(0)=7.10^{9}$ \\
\hline 2014 & 1 & $P(1)=7.10^{9} .1,02^{1}$ \\
\hline 2015 & 2 & $P(2)=7.10^{9} .1,02^{2}$ \\
\hline 2016 & 3 & $P(3)=7.10^{9} .1,02^{3}$ \\
\hline$\ldots$ & $\ldots$ & $\ldots$ \\
\hline$?$ & $\mathrm{t}$ & $P(t)=7.10^{9} .1,02^{t}$ \\
\hline
\end{tabular}

Fonte: dos autores.

Essa situação pode ser modelada por meio de uma função do tipo exponencial definida por $P(t)=7.10^{9} .1,02^{t}$, na qual $\mathrm{P}(\mathrm{t})$ representa a quantidade de habitantes no planeta em função de $\mathrm{t}$ anos (contados a partir de 2013), e o tempo t é dado em anos. Nesse caso, o domínio dessa função é dado pelos números reais positivos, mas o domínio pode também ser estendido aos números reais negativos, conforme a possível dúvida a seguir.

Possível Dúvida: Mas se 2013 é o ano inicial, como fazemos para saber a população do ano de 2012 ?

No enunciado, não se afirma que a taxa de $2 \%$ valeria para antes de 2013 , e nesse sentido, cabe ao professor deixar essa informação clara aos estudantes. No entanto, para responder a pergunta, é possível utilizar a mesma taxa, e dessa forma pode-se considerar a ordem do expoente $t$, tomando-o como $t=(-1)$, veja:

Quadro 02: estimativa para $t=(-1)$

\begin{tabular}{|c|c|c|}
\hline Ano & t (em anos) & P (população no ano t) \\
\hline 2012 & -1 & $P(0)=7.10^{9} .1,02^{-1}=\frac{7.10^{9}}{1,02} \cong 6,86.10^{9}$ \\
\hline
\end{tabular}

Fonte: dos autores.

Note que, para determinar a população de qualquer ano $y$, basta considerar 2013 como ano zero $(t=0)$ e, a partir daí pode-se considerar a seguinte igualdade:

$$
P(y)=7.10^{9} \cdot 1,02^{y-2013}
$$

Nesse caso, basta substituir o valor de $y$ na fórmula:

$$
P(y)=7.10^{9} \cdot 1,02^{y-2013},
$$


DOI: 10.20396/zet.v29i00.8661816

e dessa forma se pode estimar a população de qualquer ano $y$, considerando que a taxa pudesse ser usada tanto para antes de 2013 quanto para depois.

Também se pode utilizar a ideia de porcentagem e por meio da regra de três obter a população de 2012. Seja: $\left\{\begin{aligned} 7.10^{9} & =102 \% \\ x & =100 \%\end{aligned}\right.$

Essas informações podem ser reescritas como a igualdade de duas razões:

$$
\frac{7.10^{9}}{x}=\frac{102 \%}{100 \%}
$$

E como se obtém uma proporção, pode-se multiplicar meios por extremos, obtendo:

$$
\begin{aligned}
& 7.10^{9} .100=x .102 \\
& x=\frac{7.10^{9} .100}{102} \cong 6,86.10^{9}
\end{aligned}
$$

Logo, a população em 2012 é de aproximadamente $6,86.10^{9}$ habitantes.

\section{(5) Executando o plano}

Nosso objetivo é descobrir em quantos anos a população do planeta chegará a 28 bilhões de habitantes $\left(28.10^{9}\right)$, e para alcançá-lo basta substituir esse valor em $\mathrm{P}(\mathrm{t})$.

Possível Dúvida: Por que substituir 28 no lugar de $\mathrm{P}(\mathrm{t})$ ?

Para responder a essa questão, voltar-se-á ideia de como é determinada a igualdade, detalhando como construímos a tabela, e lembrando que a população está em função do tempo, e nesse caso, o valor desconhecido não é a população, mas sim o tempo. Portanto, é por isso que será substituído $28.10^{9}$ em $P(t)$, com o objetivo de descobrir qual será o valor de $t$. Apresenta-se a seguinte equação:

$$
\begin{gathered}
P(t)=7.10^{9} \cdot 1,02^{t}=28 \cdot 10^{9} \\
7.10^{9} .1,02^{t}=28 \cdot 10^{9} \\
7.1,02^{t}=28 \\
1,02^{t}=\frac{28}{7} \\
1,02^{t}=4
\end{gathered}
$$

Assim, tem-se como resultado uma equação exponencial, e para resolver esse tipo de equação, pode-se utilizar aproximações para descobrir a qual expoente elevar "1,02" para obter "4". Com o auxílio de uma calculadora científica, os alunos podem fazer alguns testes: 
Quadro 03: simulações para diferentes valores de t.

\begin{tabular}{|c|c|}
\hline$t$ & $1,02^{t}$ \\
\hline 0 & 1 \\
\hline 1 & 1,02 \\
\hline 2 & 1,0404 \\
\hline 3 & 1,061208 \\
\hline 4 & 1,08243216 \\
\hline 5 & 1,104080803 \\
\hline
\end{tabular}

Fonte: dos autores.

Para $t=5$, tem-se um valor ainda menor do que 2 para $1,02^{t}$, então se pode substituir valores maiores para se aproximar de 4:

Quadro 04: simulações para diferentes valores de t para se aproximar de 4.

\begin{tabular}{|c|c|}
\hline$t$ & $1,02^{t}$ \\
\hline 10 & 1,21899442 \\
\hline 20 & 1,485947396 \\
\hline 30 & 1,811361584 \\
\hline 40 & 2,208039664 \\
\hline 50 & 2,691588029 \\
\hline 60 & 3,281030788 \\
\hline 70 & 3,999558223 \\
\hline 71 & 4,079549387 \\
\hline
\end{tabular}

Fonte: dos autores.

Note que t está entre 70 e 71, mas para t $=70$ tem-se uma aproximação mais precisa, $\log _{0} t=70$ anos. Assim a população do planeta chegará a 28 bilhões em aproximadamente 70 anos. Portanto, em 2083, a Terra terá a máxima população que pode ser sustentada, considerando que não serão exploradas outras fontes de alimento.

\section{(6) Retrospecto}

Acredita-se que a resolução apresentada pelos estudantes seja por meio de aproximações, e na sequência serão retomadas as fases na resolução desse problema.

1. Leitura e compreensão do problema, analisando os dados que são relevantes para que se possa elaborar um plano para solucioná-lo.

2. Análise da taxa de crescimento da população, primeiro ano a ano, e percepção que esse crescimento ocorre de forma exponencial, permitindo ser modelado por meio de uma fórmula.

3. Construção de uma fórmula que relaciona a taxa de crescimento da população mundial, nos dando sua quantidade em função do tempo em anos. 
DOI: $10.20396 /$ zet.v29i00.8661816

4. Por meio de tentativas, pode-se aproximar o número de anos até descobrir aproximadamente em quanto tempo a população será aquela estimada anteriormente, chegando a uma solução para o problema.

Substituindo 70 no valor de $\mathrm{t}$ da fórmula, tem-se:

$$
P(70)=7 \cdot 10^{9} \cdot 1,02^{70}=7 \cdot 10^{9} \cdot 3,999558223=10^{9} \cdot 27,996907559 \cong 28 \cdot 10^{9}
$$

Dessa forma, verifica-se que em 70 anos, a população mundial será de 28 bilhões, o que significa no ano de 2083, a Terra terá a máxima população que pode ser sustentada, considerando que não serão exploradas outras fontes de alimento.

Uma vez terminada essa fase, os estudantes podem ser questionados a respeito da possibilidade de sempre realizar essas aproximações, e também sobre o fato de muitas vezes não ser tão simples de executar esses passos, afirmando que, para resolver esse problema, foi necessário um número considerável de tentativas, que podem tornar o cálculo trabalhoso e exaustivo.

Com o objetivo de simplificar o trabalho com essas operações, pode-se apresentar novos conceitos para os estudantes, que poderão agilizar os cálculos e trazer uma nova abordagem para o problema.

A seguir apresentam-se outras possíveis dúvidas que são esperadas de serem propostas pelos alunos sujeitos da aplicação desta trajetória e hipóteses sobre respostas e modos pelos quais os docentes podem conduzir as dúvidas propostas para exemplificar a qualidade do tratamento matemático dado ao problema inicial.

Possível Dúvida: os estudantes podem não compreender o conceito de logaritmos apenas com a definição, uma questão que pode surgir é: Mas qual é a relação de logaritmo com o problema que estamos tentando resolver?

Ao considerar essa questão, o professor pode aproveitar o contexto do problema e reescrever a equação exponencial: $1,02^{t}=4$, na forma de um logaritmo. No caso do problema, a base é 1,02, t representa o expoente ao qual essa base é elevada para que o resultado seja 4 . Nesse sentido, considerando a definição apresentada, tem-se:

$$
1,02^{t}=4 \quad \text { é o mesmo que } \quad a^{x}=b
$$

Como $x$ representa o expoente na segunda equação e x é o logaritmo, no caso da primeira equação o logaritmo é dado pela incógnita $t$, ou seja, se pode concluir que o logaritmo é a incógnita, que nada mais é do que o expoente da equação exponencial. Como na definição tem-se que $a^{x}=b$ é o mesmo que $\log _{a} b=x$, então, no caso do problema $1,02^{t}=4$, que podem ser reescrito como $\log _{1,02} 4=t$. Nesse caso, 1,02 é a base do 
DOI: $10.20396 /$ zet.v29i00.8661816

logaritmo, 4 é o logaritmando e té o logaritmo.

Possível Dúvida: Por que na definição de logaritmo o valor da base $\boldsymbol{a}$ deve ser positivo e diferente de 1 ? Por que o valor de $\boldsymbol{a}$ não pode ser negativo?

A base de um logaritmo representa a base de uma potência cujo expoente é o logaritmo, e o resultado obtido ao calcular essa potência é igual ao logaritmando. Quando se define que a base é maior do que zero e diferente de 1, são consideradas potências nos números reais, e a base precisa ser diferente de 1 uma vez que 1 elevado a qualquer expoente terá como resultado o próprio um ${ }^{8}$.

Possível Dúvida: Por que o valor de b deve ser positivo?

Sabe-se que $b$ representa o valor do resultado da potência $a^{x}$, logo retoma-se o estudo de potências e considera-se que, se $\mathrm{a} \in \mathrm{IR}_{+}^{*}$, então $a^{x}>0$, ou seja, o resultado de uma potência quando sua base é positiva e diferente de zero é sempre positivo. Trazendo esses conceitos para o logaritmo, sabe-se que o resultado de uma potência nada mais é do que o logaritmando, portanto, o logaritmando não pode ser igual a zero e também não pode ser negativo.

Se o professor optou por não apresentar os dados ( $\ln 1,02=0,02$ e $\ln 2=0,70$ ) no enunciado, ele pode utilizar uma calculadora com os alunos e utilizar as propriedades necessárias para resolver o problema, incluindo propriedade da mudança de base.

Assim, em aproximadamente 70 anos, a Terra terá a máxima população que poderia ser sustentada. Com a utilização dos logaritmos, os estudantes possuem mais uma ferramenta para resolver tanto esse quanto outros problemas que possam envolver as mesmas ideias e conceitos matemáticos.

Finaliza-se aqui o recorte desta trajetória porque se julga que há elementos suficientes para a discussão que se pretende realizar na próxima seção.

\section{Uma discussão a respeito da elaboração de THAs como estratégia de formação.}

Nesta seção será realizada uma análise interpretativa e descritiva, com base nos elementos da elaboração de THA apresentada na seção anterior, a qual nos serviu de base para fomentar a discussão que se pretende promover.

Uma primeira observação a ser considerada a respeito da elaboração de uma THA é que o sujeito que lidará com ela deve ter em mente seus três principais elementos, a saber:

\footnotetext{
${ }^{8}$ Neste caso, pode-se exibir um contraexemplo para esclarecer essa ideia. 
- o objetivo de aprendizagem, que define uma direção para o planejamento do professor;

- o plano que o professor elabora com as atividades de aprendizagem;

- o processo hipotético de aprendizagem, que apresenta uma previsão de como o pensamento e a compreensão dos estudantes evoluirão no contexto das atividades de aprendizagem.

Esses três elementos fazem parte da rotina diária de planejamento docente, ainda que não sejam documentados formalmente. São tarefas comuns de o professor refletir a respeito: de quais os objetivos vai se debruçar; por meio de quais tarefas, atividades de aprendizagem e ou materiais didáticos; e sob qual estratégia metodológica e que procedimentos de ensino vai por em prática. A ação de colocar no papel esses três elementos principais por escrito faz com que o docente (em formação inicial, ou continuada, ou em exercício) reflita de modo sistematizado sobre toda a demanda necessária para uma prática bem organizada e bem sucedida em sala de aula.

No recorte da THA apresentada na seção anterior pode-se observar esses três elementos apresentados de modo articulado e que acabam por envolver muitas outras práticas e decisões do trabalho docente. A seguir são apresentados alguns exemplos que denotam, em parte, a complexidade dessa proposição:

- a escolha da estratégia metodológica de ensino, que neste caso esteve associada à Resolução de Problemas ${ }^{9}$. Como resultado da elaboração da THA apresentada, acredita-se que o planejamento e exploração de THAs por meio da Resolução de Problemas têm potencial para o ensino de matemática e para a formação de professores, pois fornece subsídios tanto teóricos como práticos para que o professor trabalhe com a matemática nessa perspectiva.

- ter em foco o objetivo principal da aula ou unidade didática. Em particular, objetiva-se introduzir conceitos de Logaritmos e suas propriedades operatórias. $\mathrm{Na}$ THA apresentada, os autores tomam o cuidado de refletir sobre o "lugar" de Logaritmos quando associado ao conceito de funções, exponenciais e logarítmicas.

- o cuidado de como articular o conteúdo desejado (logaritmos) com um problema da realidade escolhido, para que a estratégia metodológica adotada (Resolução de Problemas) pudesse ser de fato implementada. Além disso, a delimitação dos objetivos da THA apresentados corroboram com as ideias de Gómez, González e Lupiáñez (2007), os quais constituem uma estrutura de referência que delimitam as condições e os procedimentos que o professor espera desenvolver com o

\footnotetext{
${ }^{9}$ Na THA apresentada, a Resolução de Problemas foi a estratégia adotada, mas poderia ser a partir de qualquer uma das tendências em Educação Matemática.
} 
objetivo de formular suas hipóteses sobre o processo de aprendizagem dos estudantes.

- a necessidade de contextualizar a situação-problema que seria apresentada trazendo elementos "extra" matemáticos para a discussão com os alunos, de modo a garantir que eles pudessem se envolver com a atividade de aprendizagem proposta, que é sugerido pela conversa sobre recursos naturais, sua escassez e o que tem sido feito para que eles sejam aproveitados da melhor forma possível. Esta "ambientação" e "exploração do tema" vão ao encontro dos questionamentos de Simon e Tzur (2004) sobre o professor refletir a respeito do processo: "qual tarefa, atualmente disponível para os estudantes, pode ser a base para que eles consigam alcançar os objetivos de aprendizagem?”.

- a decisão sobre a organização dos grupos de estudantes em sala de aula, sobre o tempo disponibilizado para a resolução do problema e outras tarefas que acompanham "a natureza" da Resolução de Problemas enquanto estratégia metodológica, como o auxílio que o docente deve dar aos grupos e a forma de conduzir a discussão do problema para sua sistematização.

- em especial, destaca-se o potencial exercício de realizar previsões (que Simon chama de hipotéticas) sobre quais perguntas os alunos podem fazer no desenvolvimento da ação planejada, sobre como produzir reflexões sobre o conteúdo matemático em questão e, também, sobre quais respostas e conduções o professor pode dar ou fazer a partir dos questionamentos produzidos.

Em particular, a respeito dessas previsões destacadas no último tópico é que vai se desdobrando todo o processamento hipotético da ação didática, como uma estratégia de processar mentalmente e, por escrito toda a arquitetura de uma aula, ou sequência de ensino. Nesse sentido, as THAs oportunizam aos docentes em formação analisarem aspectos que delimitam suas práticas de modo articulado, pois se envolvem em um processo de reflexão e tomada de decisão sobre demandas de seu exercício profissional. Nesta perspectiva, corrobora-se com Pires (2009, p. 164) ao afirmar que "os jovens professores precisam de conhecimentos sobre os saberes dos alunos, para gerar trajetórias hipotéticas de aprendizagem e análises conceituais para que possam ensinar Matemática”.

Além disso, na perspectiva dos autores do presente artigo, a THA pode se configurar como um recurso instrumentalizador, flexível, interativo, previsor, problematizador, dinâmico. No caso da característica instrumentalizadora, pode-se ressaltar que o professor também reflete sobre como será organizada sua aula, tendo em conta quais aspectos abordar em cada etapa, quais conteúdos abordar, quais dúvidas podem surgir e como ele vai responder a essas dúvidas, quanto tempo pode durar cada etapa da aula.

O caráter previsor se faz presente ao destacar as possíveis dúvidas que os estudantes podem apresentar. O professor geralmente prevê situações que já vivenciou e dúvidas mais comuns com as quais já se deparou, conhece os estudantes e seu contexto. Cabe salientar, que 
não é possível prever todas as dúvidas com precisão, mas o trabalho de sistematização e reflexão sobre elas já fornece um leque de possíveis abordagens que o professor pode por em prática a partir dessas dúvidas.

A característica "flexível" envolve a reflexão a respeito de qual ordem o professor pode abordar os diferentes aspectos do conteúdo explorado com a tarefa que está sendo trabalhada, se essa ordem interfere ou não na aprendizagem dos alunos ou se o professor pode invertê-la, dependendo de como os alunos respondem à tarefa. $\mathrm{O}$ professor pode conduzir a exploração do problema a partir do que os alunos interpretam do enunciado, e considerar as justificativas e argumentações deles para introduzir o conteúdo a ser explorado. Além disso, a flexibilidade é considerada, pois a THA não precisa ser aplicada justamente como foi elaborada, o professor tem autonomia pra tomar diferentes decisões as quais forem necessárias ao longo da execução da ação previamente hipotetizada.

Com relação ao aspecto interativo, o professor pode promover a participação dos alunos por meio de questionamentos previamente estabelecidos pela THA, e promover também a interação entre os alunos, dependendo da forma como ele organiza a dinâmica da aula (se os alunos vão trabalhar em duplas, trios, individualmente).

Sobre o caráter problematizador pode-se considerar a intencionalidade do professor ao fazer determinados questionamentos, de forma que esses questionamentos promovam a reflexão dos estudantes a respeito da temática e tenham condições de utilizar seus conhecimentos prévios para reelaborar suas estratégias, ou ainda, reconhecer quais pontos que eles ainda têm dúvidas para que os exponham para o professor e também para os outros alunos, de forma que eles tenham condições de trabalhar juntos na condução do problema em questão.

O dinamismo da trajetória hipotética de aprendizagem possibilita que o professor repense em sua aula e possa modificar aspectos que acredita que podem ser melhorados no sentido de favorecer a aprendizagem dos estudantes.

Os argumentos apresentados envolvem alguns aspectos da formação docente que são rotineiramente mobilizados nos cursos de formação docente, seja inicial ou continuada. Mais especificamente, as ações docentes destacadas no processamento hipotético de uma THA (qualquer que seja) dizem respeito à prática rotineira de um docente em sala de aula. A ação de se debruçar sobre elas é um exercício complexo e formativo na medida em que coloca o sujeito em formação em amplo processo de reflexão.

Nesse sentido, argumenta-se que a elaboração de THAs se revela como um potencial instrumento que pode oportunizar o desenvolvimento do conhecimento de professores de matemática e de formadores de professores.

Para os professores, a elaboração de trajetórias hipotéticas de aprendizagem proporcionam momentos de reflexão a respeito de como ensinar e quais possíveis questionamentos podem surgir quando um estudante se depara pela primeira vez com um problema. Em especial, quando o problema demanda determinado conteúdo matemático que 
DOI: 10.20396/zet.v29i00.8661816

o aluno desconhece, para o qual não dispõe de formas de lidar com esse problema. Ao professor cabe então realizar seu trabalho em sala de aula para criar condições para que esse estudante consiga construir ser próprio conhecimento a partir dos conhecimentos prévios que já constituiu anteriormente.

Outro aspecto merecedor de destaque a respeito da elaboração de uma trajetória hipotética de aprendizagem é que ela representa um processo contínuo e nunca se finda, uma vez que sempre é possível surgir uma dúvida ou questionamento não previsto pelo professor em sua THA para o qual ele precise encontrar maneiras de lidar com esse questionamento no momento da aula. Esse aspecto reflete que a formação do professor também representa um processo contínuo e ele sempre precisa estudar de modo a investir em sua própria formação.

\section{Algumas considerações}

Cabe observar que foram apresentados argumentos sobre o trabalho docente, todavia, para os estudantes, o trabalho com a Resolução de Problemas que foi apresentado na THA em tela e por meio da implementação de outras THA pode proporcionar mais autonomia, uma vez que o estudante é quem lida com um problema que para ele geralmente é inédito e diferente daqueles que ele já resolveu, e ele precisa buscar uma forma de resolvê-lo considerando seus conhecimentos prévios. Ao realizar esse trabalho, o estudante passa a compreender o seu papel em sala de aula enquanto construtor de seu próprio conhecimento e tende a tornar-se mais crítico porque pode se avaliar enquanto estudante ao perceber se ainda possui ou não dificuldades com outro determinado conteúdo que já estudou anteriormente.

A Trajetória Hipotética de Aprendizagem permite que o professor elabore suposições sobre o que estará prestes a ensinar, possibilita que por meio das atividades de aprendizagem, dos seus objetivos e dos processos hipotéticos, ele trace uma trajetória hipotética sobre a sua atuação em determinados momentos, pensando assim nas dúvidas que pode encontrar, naquilo que ele acredita que o aluno sabe, em como eles podem reagir e assim por diante. Essa perspectiva vai ao encontro das ideias de Simon (1995) quando justifica o uso do termo "hipotético", pois o professor não tem acesso direto ao conhecimento dos estudantes, mas pode inferir a natureza da compreensão que eles fazem a partir das interpretações dos comportamentos que apresentam. Ao se deparar com a realidade, o professor pode constatar se suas expectativas foram atendidas, podendo elaborar novamente suas hipóteses e criando assim um ciclo que não se encerra e que coloca o professor em constante aperfeiçoamento de sua prática.

O movimento de articulação dos três elementos centrais da THA propostos por Simon (1995), além de ser próprio da tarefa docente, pode apresentar-se como uma ação de vislumbrar o processo de ensino consonante ao de aprendizagem. A riqueza dessa articulação não reside nos elementos em si, mas sim nos movimentos entre eles, na coerência, no atendimento à concepção de uma prática voltada para a construção de competências, no poder argumentador que as hipóteses podem suscitar. 
DOI: $10.20396 /$ zet.v29i00.8661816

Vale ainda considerar que um professor também é pesquisador, pois está sempre supondo, constatando e se modificando, e neste sentido a elaboração de Trajetórias Hipotéticas de Aprendizagem pode ser um meio para o professor gerir sua formação continuada.

Em futuros trabalhos, acredita-se que seja pertinente a elaboração de outras THAs que abordem diferentes conteúdos matemáticos, com diversificadas estratégias metodológicas, com o objetivo de proporcionar alternativas para que os professores implementem em suas aulas, fazendo as devidas adaptações, já que elas não representam modelos a serem seguidos, mas sim uma possibilidade para aulas de matemática.

\section{Referências}

Angiolin, A. G. (2009). Trajetórias Hipotéticas de Aprendizagem sobre Funções Exponenciais. Dissertação de Mestrado Profissional em Ensino de Matemática. São Paulo: Pontifícia Universidade Católica de São Paulo.

Ball, D. L., \& Bass, H. (2003). Toward practice-based theory of mathematical knowledge for teaching. In B. Davis \& E. Smith (Eds), Proceedings of the 2002 Annual Meeting of the Canadian Mathematics Education Study Group (pp.3-14). Edmonton. Proceedings... Edmonton: CMESG/GCEDM.

Ball, D., Thames, M. H., \& Phelps, G. C (2008). Content Knowledge for Teaching: What make it special? Journal of Teacher Education, 59 (5), 389-407.

Barbosa, A. A. (2009). Trajetórias Hipotéticas de Aprendizagem relacionadas às razões e às funções trigonométricas, visando uma perspectiva construtivista. Dissertação de Mestrado Profissional em Ensino de Matemática. São Paulo: Pontifícia Universidade Católica de São Paulo.

Brousseau, G. (1987). Les differents roles du maitre. Colloque des P.E.N. Angers. In S. A. Martin (Ed.), Reconstructing mathematics pedagogy from a constructivist perspective. Washington, DC: National Science Foundation.

Gómez, P., González, M. J., \& Lupiáñez, J. L (2007). Adapting the Hypothetical Learning Trajectory Notion to Secondary Preservice Teacher Training. Chipre: Universidade de Chipre.

Ivars, P., Fernández, C., Llinares, S., \& Choy, B. H. (2018). Enhancing Noticing: Using a Hypothetical Learning Trajectory to Improve Pre-service Primary Teachers' Professional Discourse. Eurasia Journal of Mathematics, Science and Technology Education, 14(11), em1599. doi.org/10.29333/ejmste/93421

Lima, P. O. (2009). Uma Trajetória Hipotética de Aprendizagem sobre Funções Logarítmicas. Dissertação de Mestrado Profissional em Ensino de Matemática. São Paulo: Pontifícia Universidade Católica de São Paulo.

Luna, M. F. A. (2009). Estudo das Trajetórias Hipotéticas de Aprendizagem de Geometria Espacial para o Ensino Médio na perspectiva construtivista. Dissertação de Mestrado Profissional em Ensino de Matemática. São Paulo: Pontifícia Universidade Católica de São Paulo. 
Menotti, R. M. (2014). Frações e suas operações: Resolução de Problemas em uma Trajetória Hipotética de Aprendizagem. Dissertação de Mestrado Profissional em Matemática. Londrina: Universidade Estadual de Londrina.

Mesquita, M. A. N. (2009). Ensinar e Aprender funções polinomiais do $2^{\circ}$ grau no Ensino Médio: construindo trajetórias. Dissertação de Mestrado Profissional em Ensino de Matemática. São Paulo: Pontifícia Universidade Católica de São Paulo.

Oliveira, J. C. R (2015). Uma Trajetória Hipotética de Aprendizagem para o Ensino de Logaritmos na Perspectiva da Resolução de Problemas. Dissertação de Mestrado Profissional em Matemática em Rede Nacional. Universidade Estadual de Londrina, Londrina.

Oliveira, J. C. R., Frias, R. T., \& Omodei, L. B. C. (2014). Uma Trajetória Hipotética de Aprendizagem para o Ensino de Função Afim em um curso de Formação Continuada. Anais do Encontro Paranaense de Educação Matemática. Campo Mourão: Unespar.

Onuchic, L. R., \& Allevato, N. S.G. (2011). Pesquisa em Resolução de Problemas: caminhos, avanços e novas perspectivas. BOLEMA - Boletim de Educação Matemática, 25 (41), 7398.. Disponível em: < http://www.redalyc.org/pdf/2912/291223514005.pdf> . Acesso em: 15 jan. 2015.

Pires, C. M. C (2009). Perspectivas construtivistas e organizações curriculares: um encontro com as formulações de Martin Simon. Educação Matemática Pesquisa, 11 (1), 145-166.

Polya, G.(1994). A Arte de Resolver Problemas. Rio de Janeiro: Interciência.

Rosenbaum, L. S. (2010). Uma Trajetória Hipotética de Aprendizagem sobre funções trigonométricas numa perspectiva construtivista. Dissertação de Mestrado Profissional em Ensino de Matemática. São Paulo: Pontifícia Universidade Católica de São Paulo.

Simon, M. A (1995). Reconstructing Mathematics Pedagogy from a Constructivist Perspective. Journal for research in Mathematics Education, 26 (2), 114-145.

Simon, M. A., \& Tzur, R. (2004). Explicating the role of mathematical tasks in conceptual learning: an elaboration of the hypothetical learning trajectory. Mathematical Thinking and Learning, 6 (2), 91-104.

Steffe, L. P. (2004) On the Construction of Learning Trajectories of Children: The Case of Commensurate Fractions. Mathematical Thinking and Learning, 6 (2), 129-162.

Sztajn, P., Confrey, J., Wilson, P. H., \& Edgington, C. (2012). Learning Trajectory Based Instruction: Toward a Theory of Teaching. Educational Researcher, 41(5), 147-156.

Wilson, P. H., Sztajn, P., Edgington, C., \& Confrey, J. (2014). Teachers' use of their mathematical knowledge for teaching in learning a mathematics learning trajectory. Journal of Mathematics Teacher Education. 17, 149-175. DOI 10.1007/s10857-0139256-1

Wilson, P. H., Sztajn, P., Edgington, C., Webb, J., \& Myers, M. (2017). Changes in Teachers' Discourse About Students in a Professional Development on Learning Trajectories. American Educational Research Journal, 54(3), 568-604. DOI $10.3102 / 0002831217693801$ 